\title{
Attitude and Vibration Control System Modeling and Simulation For a Spacecraft Using Smart Materials
}

\author{
Bassam A. Albassam
}

\begin{abstract}
This paper is concerned with designing a control law for vibration suppression of a flexible spacecraft in the presence of external disturbances using distributed piezoelectric sensors and actuators. To satisfy pointing requirements and simultaneously suppress vibrations, two separate control actions are applied. The first action uses piezoceramics as sensors and actuators to actively suppress certain flexible modes through feedback control. The second control action uses open loop controller designed based on time optimal control theory to achieve fast maneuver. The two controllers has the ability to perform, simultaneously, minimum time maneuver and suppress the vibrations resulting from the bang-bang control spillover to the flexible modes. Modeling and simulation studies for the proposed control strategy on a flexible spacecraft have been carried out which demonstrate the effectiveness of the proposed approach.
\end{abstract}

Keywords-Flexible spacecraft; vibrations; controls; piezoelectric materials; sensors and actuators.

\section{INTRODUCTION}

In recent years, there has been a considerable interest in modelling and control of flexible structures. This is due to the use of lightweight materials for the purposes of speed and fuel efficiency. Furthermore, many applications, such as robotic manipulators, disk drive heads and pointing systems in space, are required to maneuver as quickly as possible without significant structural vibrations during and/or after a maneuver.

The time-optimal control for general maneuvers and general flexible structures has posed a challenging problem and is still an open area for research. In particular, the timeoptimal control for rest-to-rest slewing maneuvers of flexible structures has been an active area of research, and only limited solutions have been reported in the literature. Solution to the time-optimal control problem of a general flexible system is faced with two main obstacles. First, the number of control switching times is unknown a priori and must be guessed. Second, as the number of modes included in the model is increased, the computer time required by these numerical techniques becomes prohibitive. In the recent literature, many researchers (see, e.g., Refs. [1-4]) and many others listed in the review paper in Ref. [5] have developed computational techniques that deal with solving

Bassam A. Albassam

Mechanical Engineering Department

College of Engineering, King Saud University

P.O. Box 800 - Riyadh 11421 - Saudi Arabia time optimal control of flexible structures. In all of these published works, the exact time-optimal control input, which is of the bang-bang type, is calculated. From an implementation point of view, the bang-bang type of control can easily excite the higher order modes that are neglected in the model. Other researchers have utilized the simplicity of the time-optimal control design for the rigid body mode to design a near-minimum- time control for the flexible structure. The main difficulty in applying the minimum-time control input that is based on only the rigidbody mode while neglecting all the flexible modes, is the vibration that takes place during and after the maneuver as a result of control spillover to these modes. This has led many researchers to modify the bang-bang control input for the rigid-body mode in such a way so as not to excite the flexible modes and, therefore, reduce the vibrations. Junkins et al. [6] and Hecht and Junkins [7] have used an approximation function for the bang-bang control input with the objective of eliminating the instantaneous transition of control magnitudes at a switching time, resulting in a smoother control input. Hurtado and Junkins [8] have used soft constraints in the performance measure that penalize both the weighted combination of elapsed time and the first time-derivative of the control input resulting in a smooth near-minimum-time control input. In a similar approach, Albassam [9] has modified the time-optimal control problem for the rigid-body mode by adding hard constraints on the first and second time derivative of the control input to eliminate the sudden transition of control magnitudes at a switching time, thereby resulting in a variety of smooth control functions that minimize the energy transfer to the flexible modes. Recently, many researchers [10-12] have added point masses to a beam driven by a harmonic external excitation to either confine or completely eliminate the beam vibrations. These added masses can be thought of as simple reactions that provide transverse forces to the beam.

One promising method for actively suppressing the induced vibrations, resulting from the bang-band control action, is to use piezoelectric materials as actuators since piezoelectric materials have the advantages of high stiffness, light weight, low power consumption and easy implementation. A wide range of approaches have been proposed for using piezoelectric material to actively control the vibration of flexible structures, such as positive position feedback (PPF) [13-15] and velocity feedback [16], and so on. 
Proc. of the Third Intl. Conf. on Advances in Mechanical and Automation Engineering - MAE 2015. Copyright (C) Institute of Research Engineers and Doctors, USA .All rights reserved.

ISBN: 978-1-63248-080-4 doi: 10.15224/ 978-1-63248-080-4-71

This paper is concerned with the design of a minimum-time control input for a flexible structure with one rigid-body mode and many flexible modes to perform a quick desired maneuver. The objective is to perform a specified maneuver in minimum time while reducing any vibrations during and at the end of the maneuver. The time-optimal control design is based on only the rigid-body mode. This is motivated by the fact that the solution for the minimum-time control design for the rigid-body mode is easy to calculate and results in the minimum-time solution among the solutions when any of the flexible mode(s) are added to the model. Time-optimal control for the rigid body mode results in a bang-bang type of control action that excites the flexible modes. To, simultaneously, suppress the resulting vibrations, position and velocity feedback control action is applied using piezoelectric sensors and actuators mounted along the flexible beam. Numerical simulations performed on a model of the spacecraft with flexible appendage during rest-to-rest maneuver demonstrate the effectiveness and feasibility of the method.

The rest of the paper is organized as follows: Section 2 presents the model of spacecraft with flexible appendages bonded PZT, while Section 3 lay out the design for the bang-bang time optimal control input for the flexible spacecraft. Section 4 presents positive position feedback control using the piezoelelctric sensors and actuators to suppress the vibrations during the maneuver. Simulation example of the proposed control algorithm on a flexible spacecraft, followed by conclusions is given in Section 5.

\section{MATHEMATICAL MODELING}

Figure 1 shows the model of the flexible spacecraft under consideration. It consists of a rigid hub with radius $b$, a uniform cantilever flexible beam with surface bonded piezoelectric sensors and actuators, the length $l$ and the tip mass $\mathrm{m}_{\mathrm{t}}$. Coordinates OXY and oxy represents the inertial frame and the frame fixed on hub, respectively. The flexible deformation at point $\mathrm{x}$ with respect to the oxy frame is represented by $w(x, t)$, where $t$ denotes time and $x \in[0, l]$ the beam coordinate. The attitude angle $\theta$ denotes the relative motion of these frames, and $T_{h}$ is the control torque applied to the hub.

For system modeling, the following assumptions are made:

(i) the beam is considered to be an Euler-Bernoulli beam and the axial deformation is neglected

(ii) the piezoelectric layer is homogeneous and is uniaxially polarized;

(iii) the piezoelectric material is perfectly bonded to the beam;

(iv) the mass of the PZT does not contribute significantly to the stiffness of the beam;

(v) the gravitational effect is neglected for simplicity.

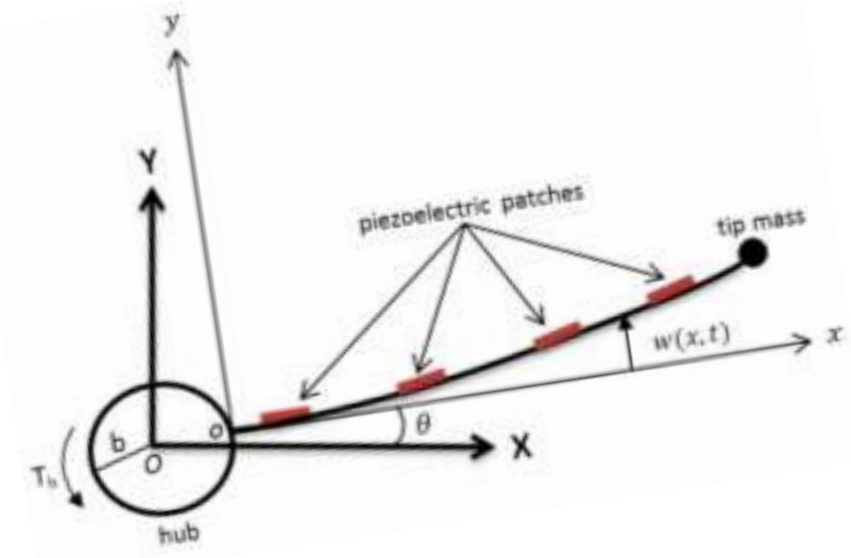

Figure 1. Model of spacecraft with flexible appendages with bonded piezoelectric material.

\section{A. Electro-mechanical Constitutive Equation}

The constitutive equation for a piezoelectric element can be written, using IEEE standard notation[17], as

$$
\begin{aligned}
& D_{3}=\varepsilon_{3}^{T} E_{3}+d_{31} T_{1} \\
& S_{1}=d_{31} E_{3}+s_{11}^{E} T_{1}
\end{aligned}
$$

where $D_{3}$ is the electric displacement along the axis in $\mathrm{C} / \mathrm{m}^{2}, E_{3}$ is the applied electrical field density in $\mathrm{V} / \mathrm{m}, S_{1}$ is the strain in $\mathrm{m} / \mathrm{m}, T_{1}$ is the stress in $\mathrm{N} / \mathrm{m}^{2}, \varepsilon_{3}^{T}$ is the permittivity of the piezoelectric material at constant stress in farad $/ \mathrm{m}, d_{31}$ is the piezoelectric charge constants in $\mathrm{C} / \mathrm{N}$, and $s_{11}^{E}$ is the elastic constants of the piezoelectric material under a constant electric field in $\mathrm{m}^{2} / \mathrm{N}$. Direction $x, y$, or $z$ is represented by the subscript 1,2 , or 3 , respectively.

To calculate the work done by the piezoelectric patches, we can rewrite equations (1) and (2) as:

$$
\begin{aligned}
& D_{3}=\left(\varepsilon_{3}^{T}-d_{31}^{2} E_{p}\right) E_{3}+d_{31} E_{p} S_{1} \\
& T_{1}=-d_{31} E_{p} E_{3}+E_{p} S_{1}
\end{aligned}
$$

where $E_{p}=\frac{1}{s_{11}^{E}}$ is defined as the short circuit modulus of elasticity for the piezoelectric material in the direction of the poling axis. Equations (3) and (4) will be used for the electro-mechanical coupling.

\section{B. Equations of Motion}

The derivation in this section follows that in Ref. [18] and repeated here for completeness. Using the assumed mode model expansion for the beam vibration analysis, the elastic deflection, $w(x, t)$, can be represented as:

$$
w(x, t)=\sum_{j=1}^{n} \phi_{j}(x) q_{j}(t)=\Phi q
$$


where $\Phi=\left[\begin{array}{llll}\phi_{1} & \phi_{2} & \ldots & \phi_{n}\end{array}\right]$ is $1 \times \mathrm{n}$ vector containing the assumed mode shapes and $q=\left[\begin{array}{llll}q_{1} & q_{2} & \ldots & q_{n}\end{array}\right]^{T}$ is $\mathrm{n} \times 1$ vector containing the generalized coordinates.

Using the extended Hamilton's principle, the equations of motion for the flexible spacecraft with surface mounted PZT sensors and actuators can be obtained from the equation:

$$
\int_{t_{1}}^{t_{2}}(\delta T+\delta W) d t=0
$$

where $\delta T$ and $\delta W$ are the virtual variation of the kinetic energy and work, respectively. $T$ is the total kinetic energy of the system and can be expressed as,

$$
T=T_{b}+\sum_{i=1}^{n_{p}} T_{p i}
$$

where $T_{b}$ represents the kinetic energy of the substructure, $T_{p i}$ represents the kinetic energy of the $i$-th PZT patch, and $n_{p}$ is the number of PZT patches. The kinetic energy is calculated from the expressions,

$$
T_{b}=T_{\text {hub }}+T_{\text {beam }}+T_{\text {tip mass }}
$$

Where $T_{\text {hub }}, T_{\text {beam }}$, and $T_{\text {tip mass }}$ are given by,

$$
\begin{gathered}
T_{h u b}=\frac{1}{2} I_{h} \dot{\theta}^{2} \\
T_{\text {beam }}=\frac{1}{2} \int_{0}^{l} \rho_{b}\left[w(x, t)^{2} \dot{\theta}^{2}+\{\dot{w}(x, t)+\dot{\theta}(b+x)\}^{2}\right] d x \\
T_{\text {tip }}=\frac{1}{2} m_{t}\left[w^{2}(l, t) \dot{\theta}^{2}+\{\dot{w}(l, t)+\dot{\theta}(b+l)\}^{2}\right] \\
T_{p i}=\frac{1}{2} \int_{x_{i}}^{x_{i}+h_{i}} \rho_{p i}\left[w^{2}(x, t) \dot{\theta}^{2}+\{\dot{w}(x, t)+\dot{\theta}(b+x)\}^{2}\right] d x
\end{gathered}
$$

where $I_{h}$ is the hub moment of inertia, $l$ the length of the flexible beam, $\rho_{b}$ the mass per unit length of the beam, $\rho_{p i}$ the mass per unit length of the $i$-th PZT patch, $x_{i}$ the starting $x$ - coordinate of the PZT patch, and $h_{i}$ the length of the PZT patch.

By defining

$$
\begin{aligned}
& J=I_{h}+\int_{0}^{l} \rho_{b}(b+x)^{2} d x+m_{t}(b+l)^{2}+\sum_{i=1}^{n_{p}} \int_{x_{i}}^{x_{i}+h_{i}} \rho_{p i}(b+x)^{2} d x \\
& M=\int_{0}^{l} \rho_{b} \Phi^{T} \Phi d x+m_{t} \Phi(l)^{T} \Phi(l)+\sum_{i=1}^{n_{p}} \int_{x_{i}}^{x_{i}+h_{i}} \rho_{p i} \Phi^{T} \Phi d x \\
& \tilde{\Phi}=\int_{0}^{l} \rho_{b}(b+x) \Phi d x+m_{t}(b+l) \Phi(l)+\sum_{i=1}^{n_{p}} \int_{x_{i}}^{x_{i}+h_{i}} \rho_{p i}(b+x) \Phi d x
\end{aligned}
$$

one can obtain an expression for the kinetic energy as:

$$
T=\frac{1}{2} J \dot{\theta}^{2}+\frac{1}{2} \dot{\theta}^{2} q^{T} M q+\dot{\theta} \tilde{\Phi} \dot{q}+\frac{1}{2} \dot{q}^{T} M \dot{q}
$$

Here, the total work of the system $W$ can be given by

$$
W=W_{b}+\sum_{i=1}^{n_{p}} W_{p i}+W_{m}
$$

where $W_{b}$ is the work done by the beam substructure, $W_{p i}$ is the work done by the $i$-th PZT patch, and $W_{m}$ is the work done by the external torque. The work done by the beam can be expressed as:

$$
W_{b}=-\frac{1}{2} \int_{0}^{l} E_{b} I_{b}\left(\frac{\partial^{2} w}{\partial x^{2}}\right)^{2} d x=-\frac{1}{2} q^{T} K_{b} q
$$

where $E_{b}$ is the modulus of elasticity for the beam, $I_{b}$ is the mass moment of inertia for the beam substructure, and $K_{b}$ is the beam stiffness matrix given by:

$$
K_{b}=\int_{0}^{l} E_{b} I_{b} \Phi^{\prime \prime T} \Phi^{\prime \prime} d x
$$

The work done by the $i$-th PZT patch is the sum of the conservative and non-conservative work terms defined as an integral over the volume of the PZT patches, given by:

$$
W_{p i}=\left(W_{p i}\right)_{c}+\left(W_{p i}\right)_{n c}=\frac{1}{2} \int_{V_{i}}\left(-T_{1 i} S_{1 i}+D_{3 i} E_{3 i}\right) d V_{i}
$$

which can be written as,

$$
W_{p i}=\frac{1}{2} w_{p i} \int_{x_{i}}^{x_{i}+h_{i}} \int_{y_{i}}^{y_{i}+t_{p i}}\left(-T_{1 i} S_{1 i}+D_{3 i} E_{3 i}\right) d y d x
$$

where $y_{i}$ is the starting point of the piezoelectric patch as measured from the neutral axis of the beam, and $w_{p i}$ is the width of the $i$-th piezoceramic wafer. Using equations (3) and (4), equation (19) can be expressed as:

$$
W_{p i}=\frac{1}{2} w_{p i} \int_{x_{i}}^{x_{i}+h_{i}} \int_{y_{i}}^{y_{i}+t_{p i}}\left\{\left(\varepsilon_{3}^{T}-d_{31}^{2} E_{p}\right) E_{3 i}^{2}+2 d_{31} E_{p} E_{3 i} S_{1 i}-E_{p} S_{1 i}^{2}\right\} d y d x
$$

Using equations (3) and (4) and assumption (iii), we can obtain

$$
W_{p i}=\frac{1}{2} \gamma_{i} v_{i}^{2}-q^{T} b_{i} v_{i}-\frac{1}{2} q^{T} K_{p i} q
$$

where $v_{i}$ is the electrode voltage and,

$$
\begin{aligned}
& \gamma_{i}=\frac{w_{p i} h_{i}}{t_{p i}}\left(\varepsilon_{3}^{T}-d_{31}^{2} E_{p}\right) \\
& v_{i}=t_{p i} E_{3 i} \\
& b_{i}=d_{31} E_{p} w_{p i}\left(y_{i}+\frac{t_{p i}}{2}\right) \int_{x_{i}}^{x_{i}+h_{i}} \Phi^{\prime \prime T} d x \\
& K_{p i}=w_{p i} t_{p i} E_{p}\left(y_{i}^{2}+y_{i} t_{p i}+\frac{t_{p i}^{2}}{3}\right) \int_{x_{i}}^{x_{i}+h_{i}} \Phi^{\prime \prime T} \Phi^{\prime \prime} d x
\end{aligned}
$$

where $t_{p i}$ is the thickness of the $i$-th PZT patch. The work done by the external torque is given by:

$$
W_{m}=T_{h} \theta
$$

Substituting Equations (16), (20), and (23) into Equation (15), one can obtain the total work as: 


$$
W=\frac{1}{2} v^{T} \bar{C} v-q^{T} \bar{B} v-\frac{1}{2} q^{T} K q+T_{h} \theta
$$

Where

$$
\begin{aligned}
& \bar{C}=\operatorname{diag}\left(\gamma_{i}\right), K=K_{b}+\sum_{i=1}^{n_{p}} K_{p i}, \\
& \bar{B}=\left[\begin{array}{llll}
b_{1} & b_{2} & \ldots & b_{n p}
\end{array}\right] \text { and } v=\left[\begin{array}{llll}
v_{1} & v_{2} & \ldots & v_{n p}
\end{array}\right]^{T} .
\end{aligned}
$$

Substituting equations (14) and (24) into equation (6), one can obtain the following equations of motion for the flexible system shown in Fig. 1:

$$
\begin{aligned}
& J \ddot{\theta}+\ddot{\theta} q^{T} M q+2 \dot{\theta} q^{T} M q+\tilde{\Phi} \ddot{q}=T_{h} \\
& \tilde{\Phi}^{T} \ddot{\theta}+M \ddot{q}+\left(K-\dot{\theta}^{2} M\right) q=-\bar{B} v \\
& \bar{C} v=\bar{B}^{T} q
\end{aligned}
$$

Equations (25) are the general nonlinear, time-varying equations of motion for the slewing structure. If the elastic displacements are small compared to the rigid body rotation, second order effects in the first equation of (25) can be neglected, resulting in a simplified model:

$$
J \ddot{\theta}+\tilde{\Phi} \ddot{q}=T_{h}
$$

On the other hand, since some of the piezoelectric patches are used as sensors and some as actuators, some will not have actuator voltage inputs and some will not have sensor voltage outputs. Therefore, $\bar{B}$ and $\bar{C}$ matrices can be decomposed into sensor and actuator parts corresponding to the sensor and actuator voltages, $v_{s}$ and $v_{a}$. Neglecting the nonlinear term, the second and third equations in (25) can be rewritten as:

$$
\begin{aligned}
& \tilde{\Phi}^{T} \ddot{\theta}+M \ddot{q}+K q=-B_{a} G_{a} v_{a} \\
& v_{s}=G_{s} C_{s}^{-1} B_{s}^{T} q
\end{aligned}
$$

where $G_{a}$ and $G_{s}$ have been added to represent the sensor and actuator amplifier gains, respectively.

In this paper, we consider a rest-to-rest attitude maneuver control problem, in which the control objective is to perform fast slewing using bang-bang control input $T_{h}$, that is, the angle $\theta(t)$ is rotated from initial state to a desired angle $\theta_{d}$ and at the same time, damp out the vibrations induced by the maneuver in the flexible element of the spacecraft using the PZT patches.

\section{BANG-BANG CONTROL INPUT DESIGN}

Equation (26) and the first equation in (27) can be put in matrix form as,

$$
m \ddot{z}+k z=a u
$$

where

$$
\begin{aligned}
& m=\left[\begin{array}{cc}
J & \widetilde{\Phi} \\
\widetilde{\Phi}^{T} & M
\end{array}\right], k=\left[\begin{array}{cc}
0 & 0 \\
0 & K
\end{array}\right], a=\left[\begin{array}{cc}
1 & 0 \\
0 & -B_{a} G_{a}
\end{array}\right], z=\left\{\begin{array}{l}
\theta \\
q
\end{array}\right\}, \\
& u=\left\{\begin{array}{l}
T_{h} \\
v_{a}
\end{array}\right\}, \text { and } a=\left[\begin{array}{cc}
1 & 0 \\
0 & -B_{a} G_{a}
\end{array}\right]
\end{aligned}
$$

and $J, \theta$, and $T_{h}$ are all scalars, $\widetilde{\Phi}$ is a $1 \times n$ vector, $M$ and $K$ are $n \times n$ matrices, $B_{a}$ is $n \times n_{a}$ matrix, $G_{a}$ is $n_{a} \times n_{a}$ matrix, and $v_{a}$ is $n_{a} \times 1$ vector.

In this section, a control input $T_{h}(t)$ is designed for the system in equation (31) to perform a rest-to-rest maneuver from the initial condition $z(0)=\left[\begin{array}{llll}0 & 0 & \ldots & 0\end{array}\right]^{\mathrm{T}}$ to the final condition $\mathrm{z}\left(\mathrm{t}_{\mathrm{f}}\right)=\left[\begin{array}{lllll}\theta_{f} & 0 & 0 & \ldots & 0\end{array}\right]^{\mathrm{T}}$ and in minimum time. From optimal control theory, it is known that the control input structure is of the bang-bang type, which can be characterized by its switching times. Many researchers (see e.g. reference [1]) have utilized this characteristic to develop numerical techniques that can transfer the timeoptimal control problem into parameter optimization problem in terms of the control switching times. They have also noted that as the number of flexible modes in the model increases, the number of control switching times also increases, thereby, making the optimal control problem more difficult, or even impossible, to solve, especially, when the number of modes becomes quite large. On the contrary, the problem becomes very simple when only the rigid-body mode is considered in the model. In this case, the control input structure is shown in Figure 4, which is a bang-bang control input that can be characterized by only one switching time $t_{s l}$ and a final time $t_{f}$.

The value of the control input $T_{h}(t)$, shown in Fig. 4, is practically constrained according to,

$$
\left(T_{h}\right)_{\min } \leq T_{h} \leq\left(T_{h}\right)_{\max }
$$

Equation (28) can be decoupled using the following transformation,

$$
z=U \eta
$$

where $U$ is a matrix with columns consisting of orthonormal eigenvectors. The decoupled equations of motion, in the modal coordinates, for the system in equation (28) becomes

$$
\begin{aligned}
& \ddot{\beta}=a_{0} T_{h} \\
& \ddot{\eta}_{i}+\omega_{i}^{2} \eta_{i}=a_{i} v_{a} \quad i=1,2, \ldots, n
\end{aligned}
$$

where $\beta(t)$ is the rigid-body coordinate, $\eta_{i}(t)$ is the $\mathrm{i}$-th modal coordinate, and $\omega_{i}$ is the $\mathrm{i}$-th frequency. The scalars $a_{i}, i=0,1, \ldots, n$, are defined by:

$$
\left[\begin{array}{cccc}
a_{0} & a_{1} & \cdots & a_{n}
\end{array}\right]^{T}=U^{T} a
$$

where $U$ is the matrix of eigenvectors.

The initial and final conditions, $\mathrm{z}(0)$ and $\mathrm{z}\left(\mathrm{t}_{\mathrm{f}}\right)$, can also be transformed to the modal coordinates as:

$$
\begin{gathered}
{\left[\begin{array}{llll}
\beta(0) & \eta_{1}(0) & \cdots & \eta_{n}(0)
\end{array}\right]^{T}=U^{T} m z(0)} \\
{\left[\begin{array}{llll}
\beta\left(t_{f}\right) & \eta_{1}\left(t_{f}\right) & \cdots & \eta_{n}\left(t_{f}\right)
\end{array}\right]^{T}=U^{T} m z\left(t_{f}\right)}
\end{gathered}
$$


Analytical solutions for the control switching and final times do exist in the literature (see e.g. reference [9]) and are given by,

$$
\begin{gathered}
t_{s 1}=\sqrt{\frac{\beta\left(t_{f}\right)}{\left(T_{h}\right)_{\max } a_{0}}} \\
t_{f}=2 t_{s 1}
\end{gathered}
$$

\section{POSITIVE POSITION FEEDBACK}

It is expected that applying the bang-bang control input torque designed in section III to the hub excites the flexible modes and results in excessive vibrations. Therefore, we apply positive positon feedback method utilizing the embedded piezoelectric sensors and actuators to suppress the vibrations and achieve precision pointing accuracy. This is done by using the positive position feedback method as an active vibration control technique. This technique, in effect, adds damping to the flexible modes that results in vibration attenuation.

Applying the coordinate transformation,

$$
q=V \delta
$$

to the system in equations (26) and (27) results in,

$$
\begin{gathered}
J \ddot{\theta}+F^{T} \ddot{\delta}=T_{h} \\
\ddot{\delta}+C_{d} \dot{\delta}+\Lambda \delta+F \ddot{\theta}=-V^{T} B_{a} G_{a} v_{a} \\
v_{s}=G_{s} C_{s}^{-1} B_{s}^{T} V \delta
\end{gathered}
$$

where $V$ is a matrix with columns consisting of orthonormal eigenvectors of $M$, and

$$
\begin{gathered}
V^{T} M V=I \\
V^{T} K V=\Lambda=\operatorname{diag}\left(\omega_{i}^{2}\right)
\end{gathered}
$$

In addition, Modal damping has been introduced to the flexible modes, where the modal damping matrix $C_{d}$ can be expressed as $C_{d}=\operatorname{diag}\left(\zeta_{i} \omega_{i}\right)$.

To facilitate the vibration compensator development using positive position feedback, and by neglecting the $F \ddot{\theta}$ term in equation (39), one can obtain the following decoupled vibration motion:

$$
\ddot{\delta}+C_{d} \dot{\delta}+\Lambda \delta=\bar{B}_{a} v_{a}
$$

where $\bar{B}_{a}=-V^{T} B_{a} G_{a}$.

The conventional PPF compensator [19] can be written in the modal co-ordinate form as follows,

$$
\ddot{\xi}+C_{f} \dot{\xi}+\Lambda_{f} \zeta=\Lambda_{f} \bar{C} \eta
$$

where $\xi$ is the compensator coordinate, $C_{f}$ is the compensator damping matrix, and $\Lambda_{f}$ is the stiffness matrix of the compensator, and $\bar{C}_{s}=G_{s} C_{s}^{-1} B_{s}^{T} V$. Considering the symmetric placement of the piezoelectric sensors and actuators, here, we assume that $\bar{C}_{s}=\bar{B}_{a}^{T}$.

Now, by choosing the control input $v_{a}=\bar{G} \Lambda \xi$ in the form of position feedback from compensator output, we arrive at the following set of system and compensator equations,

$$
\begin{aligned}
& \ddot{\delta}+C_{d} \dot{\delta}+\Lambda \delta=\bar{B}_{a} \bar{G} \Lambda \xi \\
& \ddot{\xi}+C_{f} \dot{\xi}+\Lambda_{f} \xi=\Lambda_{f} \bar{B}_{a}^{T} \delta
\end{aligned}
$$

The stability condition for the two combined systems is known to be $[13,14]$,

$$
\Lambda-\bar{B}_{a} \bar{G} \Lambda \bar{B}_{a}^{T}>0
$$

i.e., the matrix should be positive definite.

\section{NUMERICAL SIMULATIONS}

In order to demonstrate the effectiveness of the proposed control schemes, numerical simulations have been performed and presented in this section. The key technical indexes of flexible spacecraft used in the simulation are given in Table 1 and the type of PZT-5A piezoelectric ceramic plates [13] is bonded to the surface of the flexible appendage. In the simulation, the first five vibration modes have been taken into account in the model used for simulating the spacecraft at $\omega_{1}=3.14 \mathrm{rad} / \mathrm{s}, \omega_{2}=14.41$ $\mathrm{rad} / \mathrm{s}, \omega_{3}=41.17 \mathrm{rad} / \mathrm{s}, \omega_{4}=84.78 \mathrm{rad} / \mathrm{s}$, and $\omega_{5}=143.61$ $\mathrm{rad} / \mathrm{s}$ with damping $\zeta_{1}=\zeta_{2}=\zeta_{3}=\zeta_{4}=\zeta_{5}=0.0001$, respectively.

Table I. The parameters of flexible spacecraft.

\begin{tabular}{|l|c|}
\hline \multicolumn{1}{|c|}{ Parameter } & Value \\
\hline Size of the beam & $2400 \times 200 \times 3 \mathrm{~mm}$ \\
\hline Material of the beam Aluminum & Aluminum \\
\hline Weight of the tip mass & $1 \mathrm{~kg}$ \\
\hline Radius of central rigid body & $\mathrm{b}=640 \mathrm{~mm}$ \\
\hline Inertia moment of the rigid body & $11 \mathrm{~kg} \cdot \mathrm{m}^{2}$ \\
\hline
\end{tabular}

\section{DISCUSSIONS AND CONCLUSIONS}

In this paper, two controllers were designed with the objective of performing a minimum time maneuver of a rigid hub with attached flexible appendage simulating a spacecraft model. The first controller, namely, the rigid hub torquer, is designed using the time optimal control theory to perform a 90 degree maneuver and is based on only the rigid body mode alone while neglecting all the flexible modes to achieve fast maneuver. The resulting control torque is of the bang-bang type and has the advantage of performing the fastest response compared to that when any of the flexible appendages are included in the control design. The second control input is the voltage input to the piezo patch actuators, which is responsible to suppress the vibrations resulting from the bang-bang control input 
spillover to the flexible modes. The second control design is based on the positive position feedback control design method, in which the piezo sensor voltage is feedback to the piezo actuator. In the simulation, only one piezo actuator and one piezo sensor, bonded symmetrically opposite to each other, are used for this study.

As seen in Fig. 2, the bang-bang control input $T_{h}$ is able to perform the maneuver in 3.35 seconds with $\left(T_{h}\right)_{\max }=20 \mathrm{~N} . \mathrm{m}$ . It is also noticed the differences in response between the two cases with and without piezo vibration suppression. The effectiveness of this design method can also be seen in Figs. 3 and 6 which show simulations for the hub angular velocities and the first flexible modal displacement for the two cases, respectively. Fig. 4 shows the bang-bang control torque input resulting in minimum time maneuver, while Fig. 5 shows the voltage values input to the piezo actuator responsible for vibration attenuation.

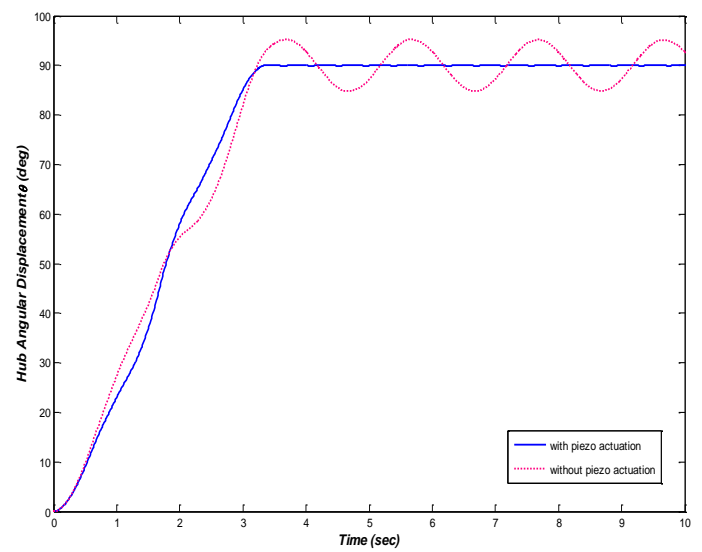

Figure 2. Time optimal hub angular displacement for two cases: with and without piezo vibration suppression.

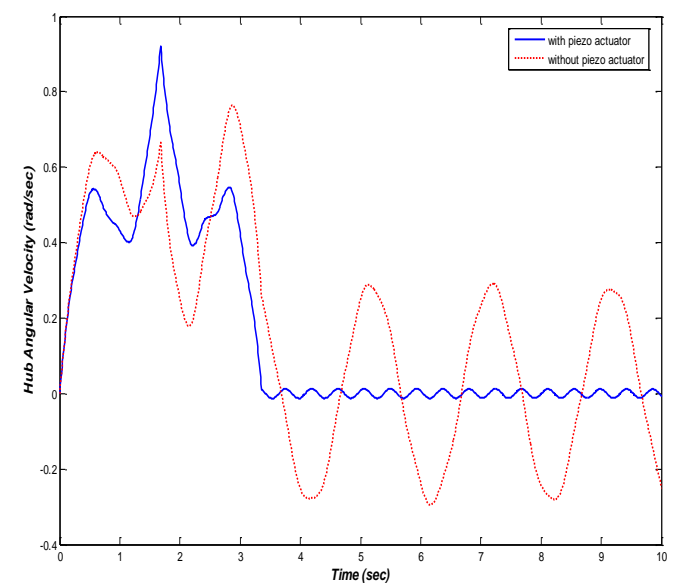

Figure 3. hub angular velocities for two cases: with and without piezo vibration suppression.

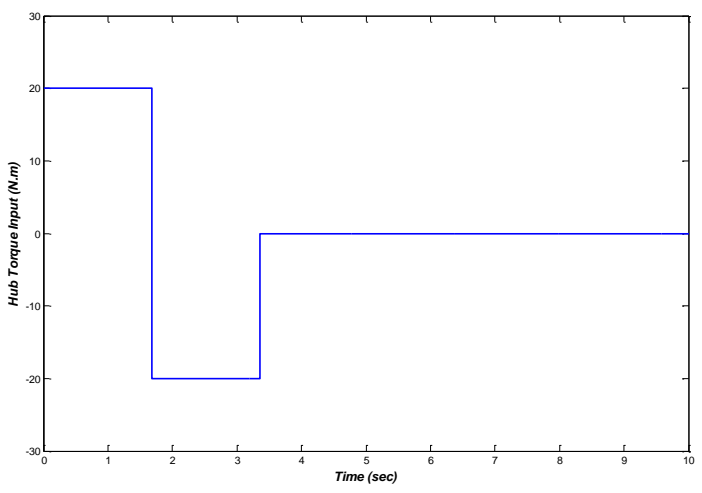

Figure 4. Time optimal hub torque input.

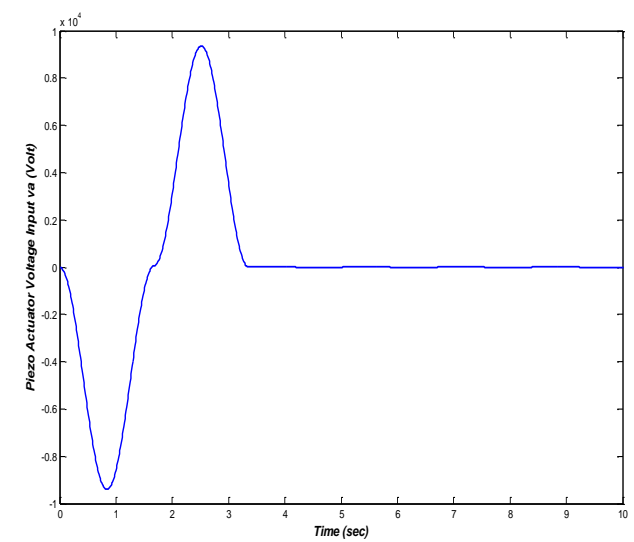

Figure 5. Voltage input to the piezo actuator patch.

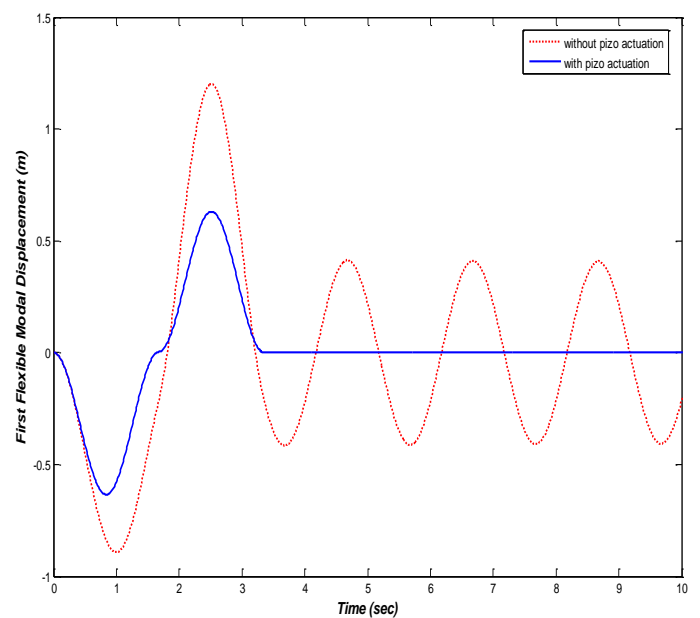

Figure 6. Simulation results for the first modal displacements for the two cases: with and without piezo vibration suppression. 


\section{References}

[1] T. Singh, S. Vadali, Robust time-optimal control: frequency domain approach, Journal of Guidance, Control, and Dynamics 17 (1994) 346-353.

[2] G. Singh, P. Kabamba, N. McClamroch, Planar, time-optimal, rest-to-rest slewing maneuvers of flexible spacecraft, Journal of Guidance, Control, and Dynamics 12 (1989) 71-81.

[3] Q. Liu, B. Wie, Robust time-optimal control of uncertain flexible spacecraft, Journal of Guidance, Control, and Dynamics 15 (1992) 597-604.

[4] J. Ben-Asher, J. Burns, E. Cliff, Time-optimal slewing of flexible spacecraft, Journal of Guidance, Control, and Dynamics 15 (1992) 360-367.

[5] S. Scrivener, R. Thompson, Survey of time-optimal attitude maneuvers, Journal of Guidance, Control, and Dynamics 17 (1994) 225-233.

[6] J.L. Junkins, Z. Rahman, H. Bang, Near-minimum-time control of distributed parameter systems: analytical and experimental results, Journal of Guidance, Control, and Dynamics 14 (1991) 406-415.

[7] N. Hecht, J.L. Junkins, Near-minimum-time control of a flexible manipulator, Journal of Guidance, Control, and Dynamics 15 (1992) 477-481.

[8] J. Hurtado, J.L. Junkins, Optimal near-minimum-time control, Journal of Guidance, Control, and Dynamics 21 (1998) 172-174.

[9] B.A. Albassam, Optimal near-minimum-time control design for flexible structures, Journal of Guidance, Control, and Dynamics 25 (2002) 618-625.

[10] R.F. Keltie, C.C. Cheng, Vibration reduction of a mass-loaded beam, Journal of Sound and Vibration 187 (1995) 213-228.

[11] Y. Chen, On the vibration of beams and rods carrying concentrated masses, Journal of Applied Mechanics 30 (1963) 310-311.

[12] L. Parnell, M. Cobble, Lateral displacement of a vibrating cantilever beam with a concentrated mass, Journal of Sound and Vibration 44 (1997) 499-511.

[13] Hu, Q. L. and Ma, G. F., 2005, "Vibration suppression of flexible spacecraft during attitude maneuvers," Journal of Guidance, Control, and Dynamics 28, 377-380.

[14] Fanson, J. L. and Caughey, T. K., 1990, "Positive position feedback control for large structure," AIAA Journal 28, 717-724.

[15] Hu, Q. L. and Ma, G. F., 2005, "Variable structure control and active vibration suppression of flexible spacecraft during attitude maneuver," Aerospace Science and Technology 9, 307-317.

[16] Tzou, H. S., 1993, Piezpelectric Shells - Distributed Sensing and Control of Continua, Kluwer Academic, London.

[17] IEEE Std 1987, IEEE Standard On Piezoelectricity, 176, 9-14.

[18] Hu, Q., Xie, L., and Gao, H., 2007 “Adaptive Variable Structure and Active Vibration Reduction for Flexible Spacecraft under Input Nonlinearity," Journal of Vibration and Control, 13, 15731602.

[19] Han, J. H., Rew, K. H., and Lee, I., 1997, “An experimental study of active vibration control of composite structures with a piezoceramic actuator and a piezo film sensor," Smart Materials and Structures, 6, 549-558. 